\title{
Criminologie
}

\section{La police et ses auxiliaires particuliers : informateurs, délateurs et agents provocateurs}

\section{Marc Laurendeau}

Volume 17, numéro 1, 1984

La police après 1984

URI : https://id.erudit.org/iderudit/017193ar

DOI : https://doi.org/10.7202/017193ar

Aller au sommaire du numéro

Éditeur(s)

Les Presses de l'Université de Montréal

ISSN

0316-0041 (imprimé)

1492-1367 (numérique)

Découvrir la revue

Citer cet article

Laurendeau, M. (1984). La police et ses auxiliaires particuliers : informateurs, délateurs et agents provocateurs. Criminologie, 17(1), 117-125.

https://doi.org/10.7202/017193ar d'utilisation que vous pouvez consulter en ligne.

https://apropos.erudit.org/fr/usagers/politique-dutilisation/ 


\section{LA POLICE ET SES AUXILIAIRES PARTICULIERS: INFORMATEURS, DELLATEURS ET AGENTS PROVOCATEURS \\ Marc Laurendeau*}

L'institution policière possède une organisation et une mission spécifique qui sont celles d'une "armée de droite»'. Elle est chargée de la mise en application quotidienne du droit. Dans son action, la police demeure elle-même soumise au respect de la loi, sans quoi elle minerait ses propres assises, saperait sa justification et détruirait sa raison d'être. Seule l'idée de justice peut faire accepter par la société cette réalité qu'est la police. La justice est l'idéologie même de la police.

Pourtant, la police doit aussi être efficace. Pour produire des arrestations importantes et obtenir des condamnations, la police est souvent amenée, en cours de route, à fermer les yeux sur l'application de la loi. La technique classique utilisée est celle des indicateurs, une méthode parfois nécessaire mais qui peut se révéler contraire au droit puisqu'elle suppose le secret de l'immunité. L'indicateur ne peut, en effet, donner des renseignements qu'à condition de rester anonyme. Cet anonymat entraîne par conséquent que non seulement le policier va cacher les sources de ses informations à l'appareil judiciaire, mais surtout, qu'il va "faire une faveur» à celui qui lui aura donné une information intéressante.

\section{UN PROCÉDÉ TRANSACTIONNEL}

Cette technique reste profondément ancrée dans la pratique policière. Dès qu'un crime est commis, les policiers enquêteurs s'empressent de rencontrer les informateurs «institutionnels», c'est-àdire ceux qui agissent dans un rôle officiel au nom d'organisations publiques et privées: concierges d'appartement, gérants d'usine, directeurs d'école, officiers municipaux, responsables de maisons de transitions, etc. Ces individus ne sont pas nécessairement tous inspirés de manière exclusive par le sens civique. Mais la police peut les amener à collaborer à des identifications et à des communications de

- Marc Laurendeau est journaliste à la télévision et dans la presse écrite. Reçu avocat en 1966, il obtint une maîtrise en science politique en 1973. Il est l'auteur du livre Les Québécois violents (Boréal-Express 1974).

1. Jean-Jacques Gleizal (1981), Figures du secret (ouvrage collectif), Presses universiaires de Grenoble, p. 235. 
renseignements par des remarques du genre: «Si jamais vous subissez un cambriolage, ne comptez pas trop sur nous je vais laisser au poste une note indiquant que vous ne voulez pas collaborer avec nous ${ }^{2}{ }^{2}$. Au cours de l'enquête s'instaure facilement un procédé transactionnel, un échange implicite.

Là où la technique devient plus délicate et plus préoccupante, c'est lorsque le policier et l'indicateur passent un contrat où des renseignements sont échangés contre la tolérance d'une forme de criminalité. Pour évoquer un exemple concret et réel ${ }^{3}$, un policier rencontre un informateur et celui-ci, disant qu'une certaine prostituée aimerait travailler pour lui, demande au policier de «faire tomber» le concubin actuel de la fille pour proxénétisme. En échange, l'informateur offre une équipe de «braqueurs». Le contrat se réalise. La brigade territoriale fait «tomber» le petit ami de la dame en question, l'indicateur récupère la fille pour son compte et l'escouade antigang arrête les trois gangsters en flagrant délit de hold-up. Tout le monde - enfin presque - est content.

La police est même amenée, de cette manière, à gérer et à utiliser des sortes de criminalité qu'elle n'arrive pas à éliminer. Elle se contente de circonscrire ces activités criminelles à des milieux précis (stratégie de "containment») et, aveu suprême d'échec, administre ces zones de criminalité par des échanges de renseignements contre l'immunité, de l'argent ou d'autres gratifications. Au lieu de contrôler le crime, la police fait alors du «crime management»" ${ }^{4}$. ${ }^{\prime}$ est ainsi que la prostitution reste, en France, le vivier des indicateurs que sont les proxénètes et que le milieu de la drogue chez nous, est devenu un nid d'informateurs. En fait, toute forme de répression du crime largement dépendante du soutien d'informateurs comporte en soi une forte dose de gérance policière du crime.

\section{QUELQUES EXEMPLES}

De 1894 à 1954, diverses commissions d'enquête (Rainville, Cannon, Coderre, Caron, etc.) sur le "vice commercialisé» à Mon-

2. Richard V. Éricson (1981), Making Crime, A Study of Butterworth, Toronto, p. 118. juillet.

3. E. Yung (1980), «Par ici les bons indices», Le Quotidien de Paris, vol. 29,

4. Jean-Paul Brodeur (1981), «Legitimizing Police Deviance», in Clifford D. Shearing, Organizational Police Deviance (ouvrage collectif), Toronto, Butterworth. 
tréal, ont démontré que la police, renonçant à éliminer totalement la prostitution, la confinait à un quartier précis et arrivait à en retirer des avantages, c'est-à-dire de l'argent et de l'information. La police cueillait ainsi des renseignements mais devenait partiellement corrompue. Plus récemment, l'effarante expansion du trafic de la drogue a amené les services policiers à utiliser systématiquement l'infiltration, les informateurs gratifiés et même à provoquer, grâce à ses agents, des transactions fictives pour prendre des trafiquants au piège. Tout ceci suppose que la police prend, pour être efficace, de très grandes libertés avec la loi et que les arrangements préférentiels, conclus quotidiennement avec les informateurs, finissent par avoir un effet corrupteur sur les policiers eux-mêmes. Ce n'est pas une coïncidence si, ces dernières années, un haut dirigeant de la GRC, chargé des stupéfiants au Québec, et le chef de l'escouade des drogues à la police de la Communauté urbaine de Montréal ont été accusés de trafic de narcotiques. Sans vouloir préjuger de la décision finale des tribunaux dans les cas individuels, on peut affirmer que les autorités à la GRC et à la police de la CUM ont perçu là une situation propice à la corruption.

Dans un autre domaine, celui du terrorisme au Québec, les services policiers, pourtant témoins de la vigueur croissante que prenait le Front de libération du Québec depuis sept ans, ne sont pas arrivés à maîtriser dans un délai raisonnable la Crise d'octobre 1970. Cette humiliation, de même que certains signaux émanant du pouvoir politique fédéral, les ont amenés à pousser plus loin l'infiltration, à utiliser occasionnellement la provocation et les tactiques de déstabilisation. En 1971, un communiqué du FLQ, rédigé par un dirigeant de la GRC, appelait les terroristes à l'action armée plutôt qu'à se joindre au Parti québécois (comme le proposait Pierre Vallières): le pouvoir est au bout du fusil, rappelait ce communiqué préparé par le surintendant Cobb de la GRC. De même, la police de la CUM avait totalement infiltré, à partir de 1971, une cellule du FLQ. Elle y possédait une informatrice, auteur de plus d'une dizaine de communiqués, participante à des actes terroristes et allant même, dans un cas de vol, jusqu'à recruter les jeunes activistes qui furent tous arrêtés.

L'escouade antiterroriste, pour certains de ces complots, fournissait de l'argent. Ả partir de mai 1972, la Section antiterroriste de la CUM, fermant les yeux sur un crime mineur mais susceptible de poursuite, recruta un second informateur dans le groupe. Ce même informateur fut subséquemment utilisé pour faire de l'obstruction aux travaux de la Commission Keable en 1979. On ne peut donc que 
constater la justesse de la phrase du capitaine Julien Giguère, lorsqu'il confiait spontanément à l'enquêteur gouvernemental JeanFrançois Duchaîne, que le «FLQ, c'était nous autres».

Pour s'accréditer dans le milieu, un informateur est amené à participer à des crimes. Sinon il restera à la périphérie du groupe sans avoir accès aux plus précieux renseignements. Là se situe le principal danger de la méthode: pour obtenir une information qui soit complète et proche du foyer de l'action, l'agent sous contrôle policier doit transgresser la loi de manière grave et susceptible de créer des victimes.

\section{L'IMMUNITÉ JUDICIAIRE}

En 1982, une technique policière connexe a commencé à prendre une énorme expansion chez nous: contre rémunération, immunité judiciaire, avantages en détention et autres gratifications, un personnage du milieu criminel était persuadé de témoigner contre ses complices. Parfois le délateur était directement coauteur du crime (et, dans un cas précis, possédait à son actif une trentaine de meurtres). À d'autres occasions, il s'agissait d'un individu impliqué dans le monde interlope. La vague des délateurs a provoqué des révélations spectaculaires et des procès retentissants. Dans un de ces derniers, trois délateurs constituaient l'essentiel de la preuve.

Ce type d'arrangement préférentiel existe aux États-Unis. En garantissant, par exemple, l'immunité à Linda Kasabian, une des filles du clan Charles Manson, le District Attorney de Los Angeles réussit à la faire témoigner contre ses complices et à obtenir la condamnation (pour le meurtre de Sharon Tate) du gourou et de ses principaux acolytes. Mais, chez nos voisins américains, même si l'arrangement préférentiel s'est développé plus rapidement qu'au Canada et au Québec, il est l'objet d'un encadrement législatif. Au Québec, on s'y est dirigé tête baissée.

Depuis 1970, existe aux États-Unis le «Federal Witness Protection Program», qui permet à la police d'obtenir le témoignage de membres de la pègre contre leurs complices en garantissant aux délateurs une protection physique durant les procédures pour le témoin et sa famille, de nouveaux papiers d'identité ( $y$ compris le permis de conduire et la carte de sécurité sociale), un logement et un emploi.

\section{AUTRES BÉNÉFICES}

Depuis 1981, ce programme, qui coûte annuellement 28 millions $\$$ est l'objet de critiques sévères au Congrès et dans la presse. 
Plusieurs témoins protégés, une fois «relocalisés», ont continué à commettre de nouveaux crimes. Cet inconvénient vient évidemment du fait qu'il s'agit de criminels endurcis, dont la conversion s'est opérée contre gratification. $23 \%$ des «témoins protégés» furent accusés de crimes subséquents. Même s'il s'agit d'un taux de récidive inférieur à celui des criminels remis en liberté, le pourcentage reste impressionnant. Plusieurs commettent des vols avec violence, des meurtres parfois. Fréquemment, des témoins protégés négligent de payer leurs dettes, dès qu'ils obtiennent une nouvelle identité. Une étude a démontré que 32 témoins protégés avaient laissé 7 millions \$ en dettes que les créanciers ont peu d'espoir de se voir rembourser. Enfin, la coupure de la vie antérieure s'avère insupportable pour certains témoins protégés: le taux de suicide est cinquante fois plus élevé chez ces délateurs que dans la population en général.

Au Québec, l'expérience est plus récente mais déjà, l'on sait que le délateur Pierre McSween, rendu célèbre lors des audiences de la CECO (sur le crime organisé), en 1975, est maintenant accusé d'une longue série de fraudes présumément commises dans les années qui suivirent. Par ailleurs, le fait que le délateur-vedette Donald Lavoie ait dû admettre s'être parjuré antérieurement, lors de l'affaire Prucha (peu après son passage du côté de la police) a souvent été invoqué par les avocats de la défense. Lavoie, de plus, a admis devant le tribunal que la vengeance contre Claude Dubois était l'un des motifs de son revirement. «ll va me payer ça, je vais me venger» disait Donald Lavoie aux avocats Frank Schoofey et Gary Martin, juste avant d'amorcer son passage du côté des services policiers, de devenir transfuge.

On constate donc que la réinsertion des délateurs dans la société cause de très sérieux problèmes. Pendant la dernière décennie, nos voisins américains voulant obtenir des condamnations de dirigeants du crime organisé ont placé d'importantes ressources à la disposition des délateurs. Ceux-ci sont aujourd'hui 4286 et les ÉtatsUnis se retrouvent avec un nouveau fléau social, un autre foyer de criminalités. Par ailleurs, dans l'intérêt supérieur de la justice, quand un témoin est ma par un désir de vengeance, cette motivation doit être prise en considération par le tribunal, puisqu'elle met en péril la fidélité du témoignage. L'utilisation des délateurs peut déclencher la condamnation surprenante de personnages haut placés mais cette méthode comporte aussi des coûts. 


\section{DES PRATIQUES INCONTRÔLÉES}

C'est devenu un cliché que d'affirmer qu'une police efficace ne saurait se passer d'informateurs. Tout service policier le moindrement sophistiqué doit s'appuyer sur un réservoir d'informateurs. En 1829 , quand Robert Peel créa la police moderne britannique, il lui donna pour mission de prévenir le crime et d'appréhender les criminels grâce à une pénétration policière de la société civile. Même si, à l'époque, cette présence policière, de 24 heures par jour, devait s'effectuer en uniforme, elle souleva un débat passionné sur l'atteinte potentielle aux libertés individuelles.

Aujourd'hui, la pénétration policière de la société civile se fait souvent grâce à des policiers non identifiés ou sous fausse identité, des policiers undercover. Mais cette méthode présente des difficultés puisque les policiers ont des caractéristiques sociales et culturelles qui les font reconnaître; au sein d'un groupe homogène de contestataires politiques, par exemple, certains traits peuvent faire ressortir les policiers de manière gênante. Le moyen le plus souple et le plus flexible consiste donc à recruter un agent de la police (par opposition à un agent de police) c'est-à-dire un individu qui, durant une période à déterminer et contre une gratification à négocier, agira pour le compte du service policier. Cet informateur, libre des exigences de l'éthique et de la formation policière, sans sécurité d'emploi ni plan de pension, possèdera une marge de manœuvre plus considérable.

Cette souplesse prend évidemment place aux dépens d'un contrôle de la légalité et de l'opportunité des gestes à poser par l'informateur. Pour obtenir des renseignements sur un meurtre commis par un tiers, le policier enquêteur fermera les yeux sur une série d'appels obscènes faits auparavant par la nouvelle «recrue». Dans un autre cas, le policier ne portera pas plainte contre le petit «pushen» pris en possession de marijuana, à condition que celui-ci fournisse des renseignements sur un gros trafiquant d'héroïne. Si l'informateur est rémunéré, le tarif reste arbitraire. Ainsi, beaucoup de Canadiens ont trouvé exorbitante la somme de 100000 \$ versée à Clifford Olson, ancien informateur, pour qu'il révèle divers endroits où il avait enterré ses jeunes victimes.

La gérance des informateurs reste un domaine qui échappe aux normes. Tout repose sur un chantage qui s'opère secrètement entre le policier et son informateur. L'absence de règles écrites de comportement et d'une forme de controle externe (analogue à l'autorisation pour écoute électronique) se fait terriblement sentir. Au-delà d'une 
certaine limite de temps, la présence d'informateurs dans le milieu ne sert plus à surveiller le crime mais à l'entretenir.

Ce phénomène de prolongation artificielle ressort clairement de l'après-Crise d'octobre. Il menace également le secteur de la drogue où la police, à force de multiplier les opérations d'infiltration, fournit des marchés pour la vente et l'achat, une main-d'œuvre, un soutien affectif, de l'argent aux personnages du milieu des narcotiques. La police peut alors amplifier la vague de crime qu'elle est chargée de réprimer. En payant $400 \$$ l'once de cocaïne à Aspen, au Colorado, les autorités policières ${ }^{6}$ ont accru le trafic à cet endroit.

En versant 10 millions $\$$ annuellement sur le marché de la drogue, la police fédérale américaine subventionne le crime organisé et stimule vraisemblablement la production (ainsi que le notait un enquêteur sénatorial). Quand les policiers de l'Agence fédérale du contrôle de la drogue investissent des ressources considérables (en argent et en personnel) sur leurs pistes probables et que l'enquête sur ces dernières se prolonge, les trafiquants ont le temps de causer des dommages considérables chez leurs victimes.

\section{UNE SURPRODUCTION DE DÉLITS}

Un peu comme certaines maladies sont engendrées directement par la médecine ou l'abus de médicaments, une forte criminalité tire son origine de l'existence des informateurs. Aux États-Unis, l'augmentation des taux d'homicides, chez les jeunes d'âge mineur, ces dernières années, est attribuable à la vaste augmentation des sommes consacrées par la police fédérale à l'achat de drogues. Ceci faisait croître les chances pour les jeunes de devenir informateurs et plusieurs furent tués par la suite. Au Québec, de nombreux individus du milieu de la drogue ont été éliminés par règlements de compte, au cours de la dernière décennie, parce qu'on les soupçonnait (parfois à tort) d'être devenus informateurs.

Il arrive que les informateurs fassent du zèle. Dès lors, ils poussent leur entourage à commettre des crimes. Ils peuvent être stimulés parce qu'ils savent que, si le groupe se fait prendre, ils bénéficieront de l'immunité. Parfois, ils se montrent aussi débordants d'activité pour justifier leur rôle, leur salaire, leur immunité judiciaire, face à leur contrôleur policier.

6. Garry Marx (1980), "Police Undercover Work», Journal of Urban Life, janvier, p. 415. 
Quand un informateur dépasse la simple cueillette de renseignements et cherche à influencer les actions de son groupe, il devient agent provocateur. Des pressions inhérentes au rôle d'informateur poussent celui-ci vers la provocation'. L'informateur est tenté de susciter le genre d'événements sur lesquels il recherche des renseignements. Un citoyen risque d'être ainsi pris au piège (entrapment) quant à un crime qu'il n'aurait peut-être pas commis, si la tentation ne s'était pas faite aussi insistante.

Les délateurs, assez curieusement, sont utilisés chez nous par les mêmes officiers de police qui avaient recours aux informateurs activistes et occasionnellement provocateurs après octobre 1970. Ces délateurs, habituellement recrutés au sein de la pègre, apportent à la justice une contribution à double volet: ils servent à démontrer qu'un meurtre peut être l'objet d'une sanction, même plusieurs années après sa commission. Mais les délateurs sont la preuve vivante que la police peut fermer les yeux sur une trentaine de meurtres avoués, à condition que celui qui les avoue possède une monnaie d'échange.

La situation ressemble au cas des fameux «repentis» italiens dont la conversion a pu faire condamner les leaders des Brigades rouges. Des grands criminels bénéficiaient ainsi de peines réduites parce qu'ils avaient vendu leurs camarades; par ailleurs, un "petit», ayant seulement fourni un soutien ou commis des actions mineures, écopait parce qu'il n'avait pas grand-chose à divulguer.

Même aux États-Unis, où le régime des délateurs a fait l'objet d'en encadrement législatif, on a vu surgir des problèmes graves. Ceci devrait suffire à rendre prudentes les autorités d'un pays et d'une province où l'utilisation systématique de ce genre de preuve $s$ 'est installée sans normes précises.

\section{CONCLUSION}

Les auxiliaires particuliers des policiers, c'est-à-dire les informateurs et les délateurs sont devenus une nécessité dans une société moderne. Mais on ne saurait les laisser fonctionner dans une zone sans droit, une sorte de «no man's land» juridique, où les contrôleurs policiers improvisent le jeu. Il faudrait rechercher en premier lieu des preuves qui n'obligent pas à fermer les yeux sur l'application 
de la loi pour favoriser des individus. Les preuves traditionnelles, basées sur les filatures, la balistique, la toxicologie, le témoignage d'un citoyen libre, etc., existent encore et ce n'est qu'à défaut de pouvoir en recueillir qu'on devrait s'en remettre aux auxiliaires particuliers. Ceux-ci font partie d'une nouvelle mode. Mais le vide législatif et réglementaire autour de ces auxiliaires ne résout en rien la choquante contradiction entre la nécessité opérationnelle et l'exigence d'équité voulant que la loi soit la même pour tous. 